Elsevier Editorial System(tm) for

International Journal of Marine Energy

Manuscript Draft

Manuscript Number: IJOME-D-16-00008R1

Title: Analysis of bi-directional ducted tidal turbine performance

Article Type: Research paper

Keywords: Tidal energy; ducted tidal turbines; bi-directional ducted turbines; actuator disc; computational fluid dynamics

Corresponding Author: Dr. Conor F Fleming, BE DPhil

Corresponding Author's Institution: Atkins

First Author: Conor F Fleming, BE DPhil

Order of Authors: Conor F Fleming, BE DPhil; Richard Willden, MEng PhD

Abstract: Several commercial tidal turbine designs feature axial flow rotors within bi-directional ducts. Such devices are typically intended to increase power extraction through a flow-concentrating effect, operating on flood and ebb tides without a yawing mechanism. Research focused on such devices has been limited so far, with available results indicating poor performance relative to bare rotors. This study further investigates the relative performance of bi-directional ducted tidal turbines in confined flow.

Several duct profiles are evaluated relative to unducted rotors using the Reynolds-averaged Navier-Stokes solver ANSYS Fluent. The rotor is represented as an actuator disc, which mimics the streamwise thrust of a real device but does not reproduce its swirl or additional turbulence generation. This idealised model achieves optimal energy extraction and enables fair comparison of duct geometries. Device power is reported relative to total frontal area, reflecting the fact that the overall dimension of the device will be limited by water depth. Comparisons based on rotor area show how the absolute power is increased by a duct, but that this is attributable to an increase in blockage.

The fundamental effect of a duct on a rotor, as well as the secondary effects of duct camber and thickness, are identified by analysing streamwise distributions of velocity, pressure and cross-sectional area along the rotor streamtube. Ducts are found to limit the expansion of the downstream flow, in turn restricting the pressure reduction immediately behind the rotor. This effect, in combination with the reduced volumetric flux through a ducted rotor relative to a bare rotor, results in reduced power extraction.

The effects of duct curvature and thickness on turbine performance are also examined. Where a ducted rotor is desirable, e.g. for the protection of rotor blades, a thick profile with slight curvature performs best.

Suggested Reviewers: Ian Masters

i.masters@swansea.ac.uk 
Tim O'Doherty

odoherty@cardiff.ac.uk

Allan Mason-Jones

mason-jonesa@cardiff.ac.uk

Michael Togneri

M.Togneri@swansea.ac.uk

Opposed Reviewers:

Response to Reviewers: REVIEWER 1 COMMENTS AND AUTHOR RESPONSES

Comment 1:

1. At the end of the introduction the author claims that the actuator disco model is sufficient to describe the device behavior. It would be interesting to know which rotor design was considered by the author: is it a symmetric airfoil based, purely twisted, around 50\% of the chord aligned blade? Or it is a standard rotor blade design assisted by a 180 degree pitch switch control based on water flow direction? A deeper explanation from the author is needed.

\section{Response 1:}

Deeper explanation provided: 'To make fair comparison between ducts we therefore use an actuator disc representation of the rotor. An actuator disc is an idealised, one-dimensional representation of a rotor, where thrust is applied against the flow in the axial direction by a thin disc. The power extracted from the flow by an actuator disc is simply the product of the axial thrust and the flow velocity, $\$ P=T$ T mathrm $\{$ disc u \mathrm\{disc\}\$. The simulations presented in this paper therefore represent the upper limit of power extraction by each duct, enabling comparison independently of rotor design.'

Comment 2:

2. In section 3 Computational modelling, the author claims that "secondary effects such as swirl and the shedding of coherent structures from the blades are not reproduced. These rotor--dependent effects would lead to further energy loss from the flow and obscure the comparison of duct performance.", while a few lines below he states "Unsteady flow features, such as vortex -shedding from the trailing edge of the duct, are not expected to significantly affect the flow through the duct or consequently the extracted power.". Is seems to me that these two sentences are contradicting each other. The author is required to remove the contradiction or providing a modification to this section.

Response 2:

Text altered: 'We note that the actuator disc is a steady-state rotor model, and do not expect unsteady duct-scale effects such as vortexshedding will have a significant effect on extracted power (based on preliminary simulations).

Therefore, the steady form of the RANS equations...'

Comment 3:

3. In section 3.2, on page 5, the author claims "Higher resolution grids $(\mathrm{y}+<1)$ were found to lead to impractically high cell counts, while lower resolution grids $\left(\mathrm{y}^{+}>30\right)$ did not adequately resolve the duct geometry or leading edge shear layer". As far as I know there is no 
relation between the normal to body resolution (driven by $\mathrm{y}^{+}$) and the resolution along the body curvilinear abscissa (i.e. the x spacing). We could agree on the resolution of the leading edge shear layer, but not on the duct geometry description, since this last depends only the spacing along the body curvilinear abscissa. A correction of the sentence in this direction is needed.

Response 3:

Clarified: 'while lower resolution grids $\left(\$ y^{\wedge}+>30 \$\right)$ did not adequately resolve the geometry or shear layer at the leading edge of the duct.'

Comment 4 :

4. In section 3.3 some theoretical results are cited. It would be nice to include some comments on the relationship between the confined flow theory and the more famous optimum condition by Betz. A clear dependency formula of power coefficient on the induction factor would be appreciated, so that the readers could easily check the results summarized in figure 4.

Response 4:

Clarified in the text - see section 3.3. The Betz limit of $\mathrm{CPmax}=16 / 27$ is only applicable to a rotor in unconfined flow. Garrett and Cummins show that in a confined flow the theoretical maximum power coefficient is greater due to the effect of the blockage ratio: CPmax $=16 / 27 * 1 /(1-$ $\mathrm{B})^{\wedge} 2=0.784$. The general formula of $\mathrm{CP}=\mathrm{f}(\mathrm{a})$ is a little more complicated, and is not repeated here for brevity.

Comment 5:

5. In the concluding section the author claims that "The results of this study indicate that a duct with moderate inner surface curvature and a flat external profile is preferable.". This sense could be regarded as true considering only the cases where a duct is present. By having a look at the results summarized in figure 6 and 7 one should observe that a bare turbine remains unbeaten. It would be nice to have a comment from the author concerning the advantage of the ducted configuration compared to the bare one, provided the clear increase in weight of the entire turbine.

Response 5:

The Conclusions have been restructured from paragraph 3 onwards to emphasise the better observed performance of the bare turbine, then to note potential advantages of a ducted configuration despite the lower power, and finally to report beneficial design features of a duct.

REVIEWER 2 COMMENTS AND AUTHOR RESPONSES

Comment 1:

My one significant concern is that the power coefficient of the unducted turbine, as plotted in figure 5 and discussed in line 165, is far too high. With a blockage of only 13\% it should not be possible to exceed the Betz limit by the extent you have. Either there has been an error in the calculation of power output, or the power coefficient has been normalised by something other than the unducted turbine area. If this is the case, I am not clear from eq. 4 exactly how the unducted power coefficient is calculated. This needs to be addressed before the paper can be published.

Response 1: 
Clarified in the text - see section 3.3. The Betz limit of CPmax = 16/27 is only applicable to a rotor in unconfined flow [28]. Garrett and Cummins [14] show that in a confined flow the theoretical maximum power coefficient is greater due to the effect of the blockage ratio: CPmax = $16 / 27 * 1 /(1-\mathrm{B})^{\wedge} 2=0.784$. The simulations predict a slightly higher $\mathrm{CP}$ due to the effects of turbulence as discussed. The unducted result in fig 5 is the same as that which is verified in fig 4 (section 3.3) - this is now emphasised in the text.

Comment 2:

- Line 9: "has been suggested" seems like the perfect place to put a reference. Who suggested it?

Response 2:

which has been suggested by manufacturers [1, 2] to have advantages

Comment 3 :

-Line 34: Typo: "The identified..." should presumably be "They

identified..."

Response 3:

Corrected

Comment $4:$

-Line 58: Would be helpful to add something here along the lines of:

"..., although note that, as discussed in section 3, the actual channel geometry as implemented in the simulations was axisymmetric."

Response 4:

Corrected

Comment 5:

-Lines 61--64: Is this simply to reduce the problem space or was there a technical motivation? A more explicit explanation of this choice would be helpful.

Response 5:

This was to reduce the problem space. The paragraph has been revised for greater clarity.

Comment 6 :

- Fig. 1: Perhaps I'm just being a bit dim, but I don't understand how Ducts B \& H can have the same proportion of total radius $(0.875 \mathrm{R})$ inside the duct, given the much greater interior curvature of B. This also applies to Ducts $C$ \& D. Is this mislabelled in the figure?

Response 6:

Ducts B and C were mislabelled - this has been corrected.

Comment 7 :

-Eq. 1: Is this the total momentum loss across the whole disc? Local momentum flux for purely axial flow through the disc will simply be rho* $(u$ d)^2 per unit area, and so the equation given here cannot be applie $\bar{d}$ as a momentum sink per area: in that case, $\mathrm{K}>2$ would have the rotor acting as a fan. On the other hand, if it was total disc resistance, I would expect to see some dependence on $\mathrm{R}$. Please add a more detailed explanation. 
Response 7:

Error in eq 1: corrected to read $\mathrm{M}=1 / 2 \mathrm{~K}$ rho $\mathrm{u}^{\wedge} 2 \mathrm{~A}$ - the resistance is dependent on the disc area (and radius $\mathrm{R}$ ). The paragraph introducing this equation has been revised for greater clarity. $M$ is a loss factor that is scaled to the dynamic head passing through the disc, and hence can be many times greater than the momentum passing through the disc as pressure head is removed from the flow.

Comment 8:

- Line 100: Were these domain boundaries chosen arbitrarily, based on sensitivity studies or based on previous experience? Give details.

Response 8:

These boundary locations were chosen based on previous experience and are consistent with a similar study by Shives and Crawford [6].

Comment 9:

- Line 121: How is duct drag computed?

Response 9:

Added sentence: Duct drag \$D \mathrm\{duct\}\$ is computed by the flow solver as the sum of viscous and pressure forces on the surface elements of the duct and resolved in the axial direction.

Comment 10:

- Eq. 3 \& 4: Axial velocity at the disc is called u_disc here, but u_d in eq. 1. The variable names should be made consistent throughout the paper.

Response 10:

Corrected

Comment 11:

Similarly, A ref and A_tot are used to refer to the same thing (total projected aréa of disc ${ }^{-}+$duct).

Response 11:

Corrected

Comment 12:

- Fig. 5: It would be very helpful to have a small inset showing the shapes of the different ducts in this figure, rather than needing to refer back several pages. I appreciate this might be difficult, however, so I don't think it is a necessary correction for resubmission.

Response 12:

Corrected for figs 5-7

Comment 13:

- Line 178: I think it would be better to say "local blockage" rather than "blockage ratio", to make it clear that you are not talking about the quantity $B$ defined elsewhere.

Response 13:

Removed this sentence as it is unnecessary.

Comment $14:-$

Fig. 8: It's unexpected to see the streamtube shrinking after the bare rotor - it should continue to expand as the pressure recovers to 
freestream (as we can see happening in fig 9b). If it shrinks we are seeing the fluid in the closed wake simultaneously accelarate and increase in static pressure. Is this purely due to energy diffusion across the streamtube boundary?

Response 14 :

Improved explanation in text: 'The wake stops expanding when the pressure equilibrates between the core and bypass flows (at \$x lapprox 0.8D\$). Subsequently, turbulent mixing causes the core flow to accelerate (and shrink) and the bypass flow to decelerate, with with both tending towards a uniform downstream velocity, \$u_linfty\$.'

Comment 15:

- Line 234: It would be clearer to say "While blockage caused by flow recirculation...".

Response 15 :

Rephrased text: 'While flow recirculation at the exterior surface of duct C increases its power by approximately 5\% relative to duct B (by increasing the effective blockage ratio), it has a more significant negative impact on hydrodynamic efficiency (roughly 25\% here).'

ADDITIONAL CHANGE BY AUTHORS:

Removed sentences from Section 4.1.2 'This indicates that the duct improves power extraction primarily by increasing the blockage ratio' as it is speculative. 
Atkins,

Woodcote Grove,

Ashley Rd,

Epsom

KT18 5BW

28 May 2016

The Editor,

International Journal of Marine Energy

Dear Editor,

I would like to submit a revised version of the paper "Analysis of bi-directional ducted tidal turbines", by Conor F Fleming and Richard H J Willden, for your consideration. Detailed responses to all reviewers' comments are included in this submission.

In this paper we use a Reynolds-averaged Navier Stokes equation solver to evaluate the performance of several bi-directional duct geometries for axial flow tidal turbines. We find that for constant blockage conditions, the power of an ideal rotor (modelled as an actuator disc) is limited by a bi-directional duct. This is attributed primarily to the confining effect of the duct on the flow downstream of the rotor, where expansion of the wake is limited, reducing the pressure change across the rotor. The relative effects of the camber and thickness of a bi-directional duct are also examined, and where a duct is desirable for other reasons (e.g. protection of blades, bearing of rotor) we recommend a thick profile with slight camber.

As corresponding author, I may be contacted at the email address conor.fleming@oxon.org. I hope you find this paper interesting and worthy of publication in the International Journal of Marine Energy.

Sincerely,

Conor Fleming 
Several ducts for bi-directional tidal turbines are evaluated through RANS modelling.

The ducted devices have lower power coefficients than a bare rotor at equal blockage.

The ducts were found to constrain the wake, reducing the rotor pressure drop.

Hydrodynamic efficiency is reduced by a duct due to additional viscous losses.

Power per unit rotor area can be increased through the addition of a duct. 
Comment 1 :

1. At the end of the introduction the author claims that the actuator disco model is sufficient to describe the device behavior. It would be interesting to know which rotor design was considered by the author: is it a symmetric airfoil based, purely twisted, around 50\% of the chord aligned blade? Or it is a standard rotor blade design assisted by a 180 degree pitch switch control based on water flow direction? A deeper explanation from the author is needed.

Response 1:

Deeper explanation provided: 'To make fair comparison between ducts we therefore use an actuator disc representation of the rotor. An actuator disc is an idealised, one-dimensional representation of a rotor, where thrust is applied against the flow in the axial direction by a thin disc. The power extracted from the flow by an actuator disc is simply the product of the axial thrust and the flow velocity, $\$ P=T$ T mathrm\{disc\} u_lmathrm\{disc\}\$. The simulations presented in this paper therefore represent the upper limit of power extraction by each duct, enabling comparison independently of rotor design.'

Comment 2 :

2. In section 3 Computational modelling, the author claims that

"secondary effects such as swirl and the shedding of coherent structures from the blades are not reproduced. These rotor--dependent effects would lead to further energy loss from the flow and obscure the comparison of duct performance.", while a few lines below he states "Unsteady flow features, such as vortex -shedding from the trailing edge of the duct, are not expected to significantly affect the flow through the duct or consequently the extracted power.". Is seems to me that these two sentences are contradicting each other. The author is required to remove the contradiction or providing a modification to this section.

Response 2:

Text altered: 'We note that the actuator disc is a steady-state rotor model, and do not expect unsteady duct-scale effects such as vortexshedding will have a significant effect on extracted power (based on preliminary simulations).

Therefore, the steady form of the RANS equations...'

Comment 3 :

3. In section 3.2, on page 5, the author claims "Higher resolution grids $\left(\mathrm{y}^{+}<1\right)$ were found to lead to impractically high cell counts, while lower resolution grids $\left(\mathrm{y}^{+}>30\right)$ did not adequately resolve the duct geometry or leading edge shear layer". As far as I know there is no relation between the normal to body resolution (driven by $y^{+}$) and the resolution along the body curvilinear abscissa (i.e. the x spacing). We could agree on the resolution of the leading edge shear layer, but not on the duct geometry description, since this last depends only the spacing along the body curvilinear abscissa. A correction of the sentence in this direction is needed.

Response 3:

Clarified: 'while lower resolution grids ( $\left.\$ y^{\wedge}+>30 \$\right)$ did not adequately resolve the geometry or shear layer at the leading edge of the duct.'

Comment 4 : 
4. In section 3.3 some theoretical results are cited. It would be nice to include some comments on the relationship between the confined flow theory and the more famous optimum condition by Betz. A clear dependency formula of power coefficient on the induction factor would be appreciated, so that the readers could easily check the results summarized in figure 4.

Response 4:

Clarified in the text - see section 3.3. The Betz limit of $\mathrm{CPmax}=16 / 27$ is only applicable to a rotor in unconfined flow. Garrett and Cummins show that in a confined flow the theoretical maximum power coefficient is greater due to the effect of the blockage ratio: $\mathrm{CPmax}=16 / 27 * 1 /(1-$ $\mathrm{B})^{\wedge} 2=0.784$. The general formula of $\mathrm{CP}=\mathrm{f}(\mathrm{a})$ is a little more complicated, and is not repeated here for brevity.

Comment 5:

5. In the concluding section the author claims that "The results of this study indicate that a duct with moderate inner surface curvature and a flat external profile is preferable.". This sense could be regarded as true considering only the cases where a duct is present. By having a look at the results summarized in figure 6 and 7 one should observe that a bare turbine remains unbeaten. It would be nice to have a comment from the author concerning the advantage of the ducted configuration compared to the bare one, provided the clear increase in weight of the entire turbine.

Response 5:

The Conclusions have been restructured from paragraph 3 onwards to emphasise the better observed performance of the bare turbine, then to note potential advantages of a ducted configuration despite the lower power, and finally to report beneficial design features of a duct.

\section{REVIEWER 2 COMMENTS AND AUTHOR RESPONSES}

Comment 1:

My one significant concern is that the power coefficient of the unducted turbine, as plotted in figure 5 and discussed in line 165, is far too high. With a blockage of only $13 \%$ it should not be possible to exceed the Betz limit by the extent you have. Either there has been an error in the calculation of power output, or the power coefficient has been normalised by something other than the unducted turbine area. If this is the case, I am not clear from eq. 4 exactly how the unducted power coefficient is calculated. This needs to be addressed before the paper can be published.

Response 1:

Clarified in the text - see section 3.3. The Betz limit of $\mathrm{CPmax}=16 / 27$ is only applicable to a rotor in unconfined flow [28]. Garrett and Cummins [14] show that in a confined flow the theoretical maximum power coefficient is greater due to the effect of the blockage ratio: CPmax = $16 / 27 * 1 /(1-\mathrm{B})^{\wedge} 2=0.784$. The simulations predict a slightly higher $\mathrm{CP}$ due to the effects of turbulence as discussed. The unducted result in fig 5 is the same as that which is verified in fig 4 (section 3.3) - this is now emphasised in the text.

Comment 2:

- Line 9: "has been suggested" seems like the perfect place to put a reference. Who suggested it? 
Response 2:

which has been suggested by manufacturers [1, 2] to have advantages

Comment 3:

-Line 34: Typo: "The identified..." should presumably be "They

identified..."

Response 3:

Corrected

Comment 4 :

-Line 58: Would be helpful to add something here along the lines of:

"..., although note that, as discussed in section 3, the actual channel geometry as implemented in the simulations was axisymmetric."

Response 4:

Corrected

Comment 5:

-Lines 61--64: Is this simply to reduce the problem space or was there a technical motivation? A more explicit explanation of this choice would be helpful.

Response 5:

This was to reduce the problem space. The paragraph has been revised for greater clarity.

Comment 6:

- Fig. 1: Perhaps I'm just being a bit dim, but I don't understand how Ducts $B$ \& $\mathrm{H}$ can have the same proportion of total radius $(0.875 \mathrm{R})$ inside the duct, given the much greater interior curvature of $\mathrm{B}$. This also applies to Ducts C \& D. Is this mislabelled in the figure?

Response 6:

Ducts B and C were mislabelled - this has been corrected.

Comment 7:

-Eq. 1: Is this the total momentum loss across the whole disc? Local momentum flux for purely axial flow through the disc will simply be rho* $(u$ d)^2 per unit area, and so the equation given here cannot be applie $\bar{d}$ as a momentum sink per area: in that case, $K>2$ would have the rotor acting as a fan. On the other hand, if it was total disc

resistance, I would expect to see some dependence on $\mathrm{R}$. Please add a more detailed explanation.

Response 7:

Error in eq 1: corrected to read $\mathrm{M}=1 / 2 \mathrm{~K}$ rho $\mathrm{u}^{\wedge} 2 \mathrm{~A}$ - the resistance is dependent on the disc area (and radius $\mathrm{R}$ ). The paragraph introducing this equation has been revised for greater clarity. $M$ is a loss factor that is scaled to the dynamic head passing through the disc, and hence can be many times greater than the momentum passing through the disc as pressure head is removed from the flow.

Comment 8:

- Line 100: Were these domain boundaries chosen arbitrarily, based on sensitivity studies or based on previous experience? Give details.

Response 8: 
These boundary locations were chosen based on previous experience and are consistent with a similar study by Shives and Crawford [6].

Comment 9:

- Line 121: How is duct drag computed?

Response 9:

Added sentence: Duct drag $\$ D_{-} \backslash$ mathrm\{duct\}\$ is computed by the flow solver as the sum of viscous and pressure forces on the surface elements of the duct and resolved in the axial direction.

Comment 10:

- Eq. 3 \& 4: Axial velocity at the disc is called u_disc here, but u_d in eq. 1. The variable names should be made consistent throughout the paper.

Response 10:

Corrected

Comment 11:

Similarly, A ref and A tot are used to refer to the same thing (total projected aréa of disc ${ }^{-}+$duct).

Response 11:

Corrected

Comment 12:

- Fig. 5: It would be very helpful to have a small inset showing the shapes of the different ducts in this figure, rather than needing to refer back several pages. I appreciate this might be difficult, however, so I don't think it is a necessary correction for resubmission.

Response 12:

Corrected for figs 5-7

Comment 13:

- Line 178: I think it would be better to say "local blockage" rather than "blockage ratio", to make it clear that you are not talking about the quantity $\mathrm{B}$ defined elsewhere.

Response 13:

Removed this sentence as it is unnecessary.

Comment $14:-$

Fig. 8: It's unexpected to see the streamtube shrinking after the bare rotor - it should continue to expand as the pressure recovers to freestream (as we can see happening in fig 9b). If it shrinks we are seeing the fluid in the closed wake simultaneously accelarate and increase in static pressure. Is this purely due to energy diffusion across the streamtube boundary?

Response 14:

Improved explanation in text: 'The wake stops expanding when the pressure equilibrates between the core and bypass flows (at \$x lapprox 0.8D\$). Subsequently, turbulent mixing causes the core flow to accelerate (and shrink) and the bypass flow to decelerate, with with both tending towards a uniform downstream velocity, \$u_linfty\$.'

Comment 15: 
- Line 234: It would be clearer to say "While blockage caused by flow recirculation...".

Response 15:

Rephrased text: 'While flow recirculation at the exterior surface of duct $C$ increases its power by approximately 5\% relative to duct B (by increasing the effective blockage ratio), it has a more significant negative impact on hydrodynamic efficiency (roughly 25\% here).'

\section{ADDITIONAL CHANGE BY AUTHORS:}

Removed sentences from Section 4.1.2 'This indicates that the duct improves power extraction primarily by increasing the blockage ratio' as it is speculative. 


\title{
Analysis of bi-directional ducted tidal turbine performance
}

\author{
Conor F Fleming*, Richard H J Willden \\ Department of Engineering Science, University of Oxford, Oxford, OX1 3PJ, UK
}

\begin{abstract}
Several commercial tidal turbine designs feature axial flow rotors within bi-directional ducts. Such devices are typically intended to increase power extraction through a flow-concentrating effect, operating on flood and ebb tides without a yawing mechanism. Research focused on such devices has been limited so far, with available results indicating poor performance relative to bare rotors. This study further investigates the relative performance of bi-directional ducted tidal turbines in confined flow.

Several duct profiles are evaluated relative to unducted rotors using the Reynolds-averaged Navier-Stokes solver ANSYS Fluent. The rotor is represented as an actuator disc, which mimics the streamwise thrust of a real device but does not reproduce its swirl or additional turbulence generation. This idealised model achieves optimal energy extraction and enables fair comparison of duct geometries. Device power is reported relative to total frontal area, reflecting the fact that the overall dimension of the device will be limited by water depth. Comparisons based on rotor area show how the absolute power is increased by a duct, but that this is attributable to an increase in blockage.

The fundamental effect of a duct on a rotor, as well as the secondary effects of duct camber and thickness, are identified by analysing streamwise distributions of velocity, pressure and cross-sectional area along the rotor streamtube. Ducts are found to limit the expansion of the downstream flow, in turn restricting the pressure reduction immediately behind the rotor. This effect, in combination with the reduced volumetric flux through a ducted rotor relative to a bare rotor, results in reduced power extraction.

The effects of duct curvature and thickness on turbine performance are also examined. Where a ducted rotor is
\end{abstract} desirable, e.g. for the protection of rotor blades, a thick profile with slight curvature performs best.

Keywords: Tidal energy, ducted tidal turbines, bi-directional ducted turbines, actuator disc, computational fluid dynamics

\section{Introduction}

There has been renewed focus on renewable energy in recent years due to the increasing environmental cost of energy derived from fossil fuels. Tidal currents are an attractive source of renewable energy due to their predictability and the low visual impact of tidal stream energy converters. This industry is in its infancy, with few devices in operation commercially (e.g. the Seagen turbine in Strangford Lough, Northern Ireland) and a variety of prototype designs under trial at various locations including the European Marine Energy Centre in the Orkney Islands, UK.

Turbine designs can be broadly categorised into axial flow (similar to conventional wind turbines), cross-flow (similar to Darrieus wind turbines), and oscillating types. An axial flow turbine may feature a duct, which has been suggested by manufacturers [1, 2] to have advantages over a bare rotor in terms of power generation, realignment of yawed flow and protection of rotor blades.

Uni-directional ducted turbines have attracted academic and industrial interest for many decades, notably in the experimental work by Foreman et al. [3, 4] which formed the basis for the later attempted commercialisation of a ducted wind turbine [5]. They typically feature a diffuser downstream of the rotor, intended to increase the pressure

\footnotetext{
${ }^{*}$ Corresponding author. Now at Atkins, Woodcote Grove, Epsom, KT18 5BW, UK

Email address: conor. fleming@oxon.org (Conor F Fleming)
} 
drop across the rotor and also increase the mass flux through the rotor, improving power extraction from the flow. Comprehensive reviews of research into uni-directional ducted devices are presented by Shives and Crawford [6] and Belloni [7].

Bi-directional turbines have received attention more recently in the growing tidal energy industry due to their suitability for operation in tidal currents. These turbines are symmetric about the rotor plane, and typically feature a converging-diverging nozzle which is intended to accelerate the flow through the rotor and hence increase the available kinetic energy flux. Such devices developed in the last ten years include the current Rotech Tidal Turbine by Lunar Energy [2], the OpenHydro turbine [1], the Solon turbine by Atlantis Resources Corporation (discontinued in 2013) [8] and the Clean Current tidal turbine (prior to 2013, now featuring a uni-directional duct) [9]. Despite the industrial interest in bi-directional ducted rotors, little research published to date has focused on this topic. Setoguchi et al. [10] test a range of bi-directional ducts, and examine the effect of the outer surface profile on internal flow acceleration. However a rotor is not represented in their experiments, and its presence would clearly impact the balance between the flow accelerated through the duct and the flow forced to pass around the outside of the duct. In a precursor to the present study, the authors designed a bi-directional ducted turbine based on a parametric computational study of a selection of candidate profiles [11]. The unducted rotor was found to yield a power coefficient approximately $75 \%$ higher than the best-performing ducted rotor, when power was normalised on total device area (including the duct where present) and under consistent blockage conditions (blockage is defined as the ratio of total device frontal area to the flow channel cross-sectional area). Similar findings are reported by Belloni et al. [12, 13], who simulate a ducted and an unducted device in confined flow. The power coefficient for the unducted turbine is roughly 50\% greater than that of the ducted turbine when based on device total area, although they found power density (power per unit rotor area) to increase. They identified two operating modes for bi-directional ducted turbines. At low rotor resistance, the flow remains attached throughout the device. The velocity through the rotor is significantly increased, but power remains low due to the low level of rotor thrust. As rotor resistance is increased the approaching flow is significantly diverted around the duct, leading to external separation at the duct leading edge. For the blockage conditions simulated, the external separation led to an increase in the effective blockage of the flow, which led to an increase in extracted power in accordance with the known effects of flow confinement [14]. However, if the rotor resistance is overly high, the benefit of leading edge separation is lost and too much flow is diverted around the device. The authors also note the reduced hydrodynamic efficiency of ducted turbines due to the additional thrust exerted by the duct.

This paper further explores the fluid mechanics of bi-directional ducted turbines. A series of duct profiles are devised to explore the effects of duct curvature and thickness on turbine power and hydrodynamic efficiency. Each device is simulated at a range of operating points using the Reynolds-averaged Navier-Stokes (RANS) equation solver ANSYS Fluent, following a modelling approach similar to that of Shives and Crawford [6]. Flow conditions within each duct are necessarily a function of duct geometry and as such optimal rotor geometry will be different for each duct [15]. To make fair comparison between ducts we therefore use an actuator disc representation of the rotor. An actuator disc is an idealised, one-dimensional representation of a rotor, where thrust is applied against the flow in the axial direction by a thin disc. The power extracted from the flow by an actuator disc is simply the product of the axial thrust and the flow velocity at the disc, $P=T_{\text {disc }} u_{\text {disc }}$. The simulations presented in this paper therefore represent the upper limit of power extraction by each duct, enabling comparison independently of rotor design. We identify trends in power and hydrodynamic efficiency and their causes are explained by reference to streamwise profiles of streamtube velocity, pressure and cross-sectional area.

\section{Ducted turbine design generation}

A series of duct geometries are devised within particular constraints, so that the effects of curvature and thickness may be explored. The first constraint imposed is that all devices are compared under consistent blockage conditions. Various authors have highlighted the sensitivity of turbine power and thrust to blockage [16, 17, 18], and it is essential to eliminate this effect from the comparison of duct geometries. The blockage ratio chosen for the current study is $B=0.131$. This corresponds to a fence of turbines with lateral centre-to-centre spacing of three rotor diameters $(D)$ apart laterally in water of depth $2 D$ although note that, as discussed in section 3 , the actual channel geometry as implemented in the simulations was axisymmetric. 

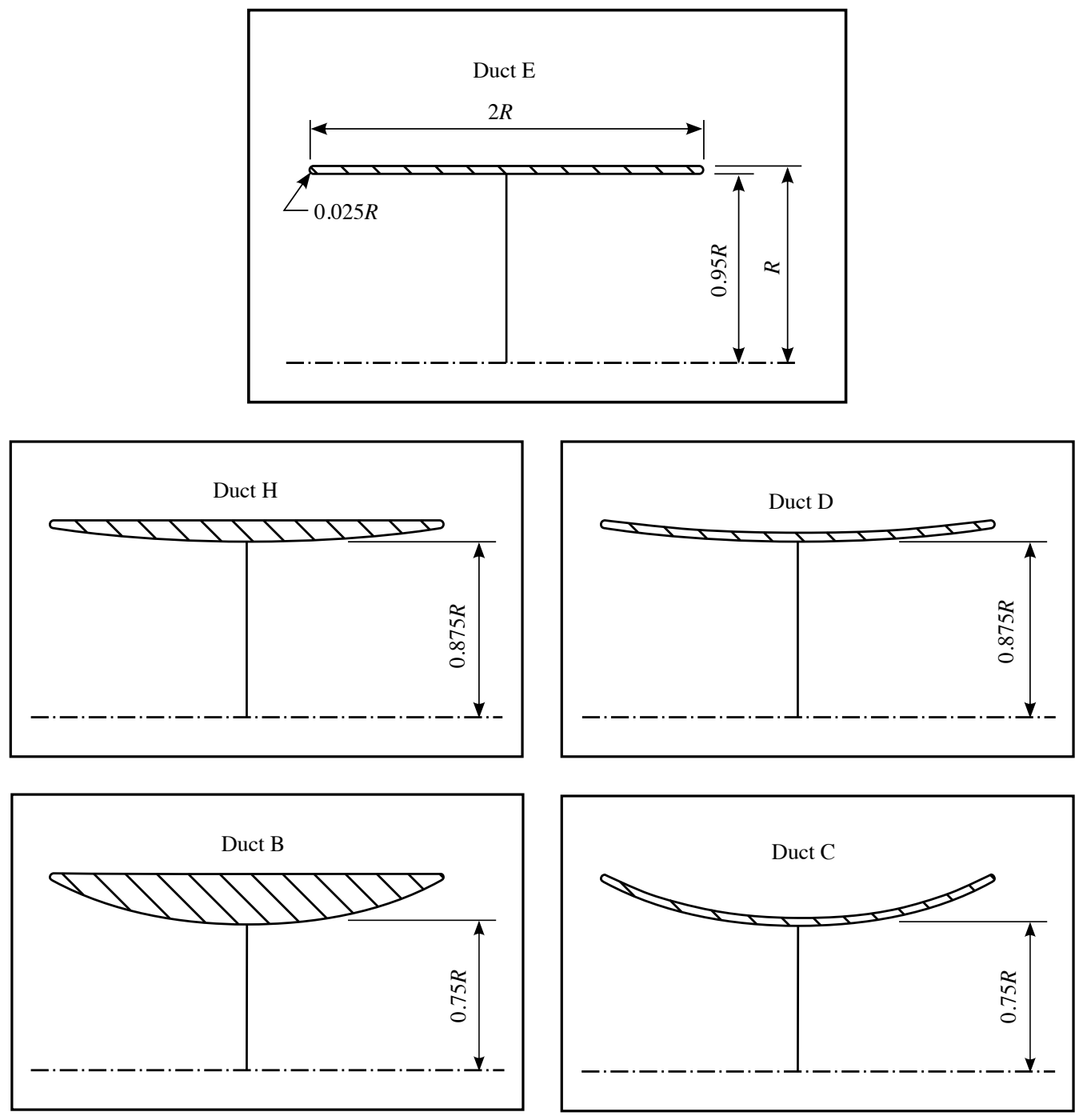

Figure 1: Profiles of ducts tested in parametric study, where $R$ is radius.

The second constraint is that the duct should be symmetric about the rotor plane to allow bi-directional operation without any yawing mechanism.

Thirdly, we limit the scope of this study to the effect of duct profile, and hence choose a single ratio of duct of duct length $L$ to diameter $D$ for all duct designs. Our choice of $L / D=1$ is a moderate value based on observations of commercial designs [1, 2, 9].

Finally, all curved surfaces are constructed from circular arcs. This is a compromise between simplicity of design and manufacture, where straight sections and corners might be preferable, and hydrodynamic considerations, where a smooth profile is favoured. Additionally, the use of simple arcs allows for the current duct geometries to be reproduced.

The candidate designs are presented in figure 1 where dimensions are given in terms of the device radius $R$. The first profile, duct $\mathrm{E}$, is produced by joining the leading and trailing edges with straight lines. Two series of designs are produced by perturbing the inner and outer surfaces of duct E. Ducts D and C are produced by joining the leading and trailing edges with concentric arcs, producing profiles of constant thickness. For ducts B and $\mathrm{H}$, the leading and trailing edges are connected by a straight outer surface, and a curved inner surface, leading to thick profiles. 


\section{Computational modelling}

\subsection{Geometry and boundary conditions}

Each device is simulated at a range of operating points to yield complete performance curves. A computationallyefficient model is developed by applying particular simplifications while still capturing the relevant flow physics.

The first simplification is to assume that the domain containing the turbine and channel is axisymmetric, which in turn requires the effects of free surface deformation, supporting structure and channel cross section to be neglected. These assumptions are not expected to affect the performance of the duct designs in relation to one another within the parametric study. Consul et al. [19] demonstrate that the effects of free surface deformation on the power of a cross-flow tidal turbine are negligible for blockage conditions of $B<0.25$. Nishino and Willden [18] investigate the influence of the aspect ratio of a rectangular channel on turbine performance, and find that it is of secondary importance to blockage.

The second simplification is to represent the rotor as an actuator disc. This idealised rotor presents a resistance in the streamwise direction only and extracts the upper limit of power from the flow. Unlike higher-order rotor modelling techniques [20, 21], secondary effects such as swirl and the shedding of coherent structures from the blades are not reproduced. These rotor-dependent effects would lead to further energy loss from the flow and obscure the comparison of duct performance. Additionally, the inclusion of rotor geometry in this comparison would require bespoke rotor design for each duct [15]. Hence, for a fair and straightforward comparison of duct performance it is preferable to assume perfect rotors in the form of actuator discs.

In the numerical actuator disc model, the resistance of the disc in the axial direction takes the form of a sink in the momentum equations, $M$,

$$
M=\frac{1}{2} K \rho A_{\mathrm{disc}} u_{\mathrm{disc}}^{2}
$$

where $\rho$ is the fluid density, $A_{\text {disc }}$ is the disc cross-sectional area, $u_{\text {disc }}$ is the local axial velocity at the disc and the momentum loss factor $K$ is a user-defined parameter which controls the magnitude of the resistance. The momentum loss factor is varied within the range $0<K<5$ to emulate the thrust of a rotor at a range of angular speeds.

We note that the actuator disc is a steady-state rotor model, and do not expect unsteady duct-scale effects such as vortex-shedding will have a significant effect on extracted power (based on preliminary simulations). Therefore, the steady form of the RANS equations is solved in a cylindrical reference frame, using the commercial code ANSYS Fluent [22]. The governing equations are discretised on a two-dimensional structured grid, which extends from $5 D$ upstream of the rotor plane to $10 \mathrm{D}$ downstream of it. These boundary locations were chosen based on previous experience and are consistent with a similar study by Shives and Crawford [6]. The outer radial boundary is set at $2.763 R$, corresponding to the desired blockage ratio of $B=0.131$.

The $k-\omega$ SST model is used for turbulence closure. This model was developed for application to flow in adverse pressure gradients, where boundary layer separation is likely to occur [23]. For the ducts studied here, adverse pressure gradients are generally expected along the exterior of the duct leading edge, as well as along the interior surface of the duct downstream of the rotor, where the cross-sectional area of the duct increases (except for duct E). Flow separation is likely to occur in these regions, and may be dependent on rotor thrust. The performance of the $k-\omega$ SST in separated flows has been evaluated extensively in the literature. Apsley and Leschziner [24] model flow through a planar diffuser with a variety of turbulence closures (linear and non-linear eddy viscosity models and differential stress models). No single turbulence model is found to be clearly superior, and of the two-equation linear eddy viscosity models examined $-k-\varepsilon, k-\omega$ and $k-\omega \mathrm{SST}-$ the $k-\omega \mathrm{SST}$ model produces the best prediction of the flow field (albeit with early separation). Shives and Crawford [6] provide a comprehensive summary of this and similar investigations of the $k-\omega$ SST model.

A uniform velocity profile is prescribed at the upstream boundary, and the Reynolds number, based on device diameter, is set to $\operatorname{Re}=4 \times 10^{7}$. This would correspond to a device of $20 \mathrm{~m}$ diameter in a flow of $2 \mathrm{~m} \mathrm{~s}^{-1}$. at the same boundary, a turbulence intensity of $T I=10 \%$ and length scale of $l=0.1 D$ are specified, following the 'high turbulence' case of Nishino and Willden [25]. At the downstream boundary, a constant pressure condition is applied. The inner radial boundary is assigned as the axis of symmetry. A slip condition is set at the outer radial boundary. 


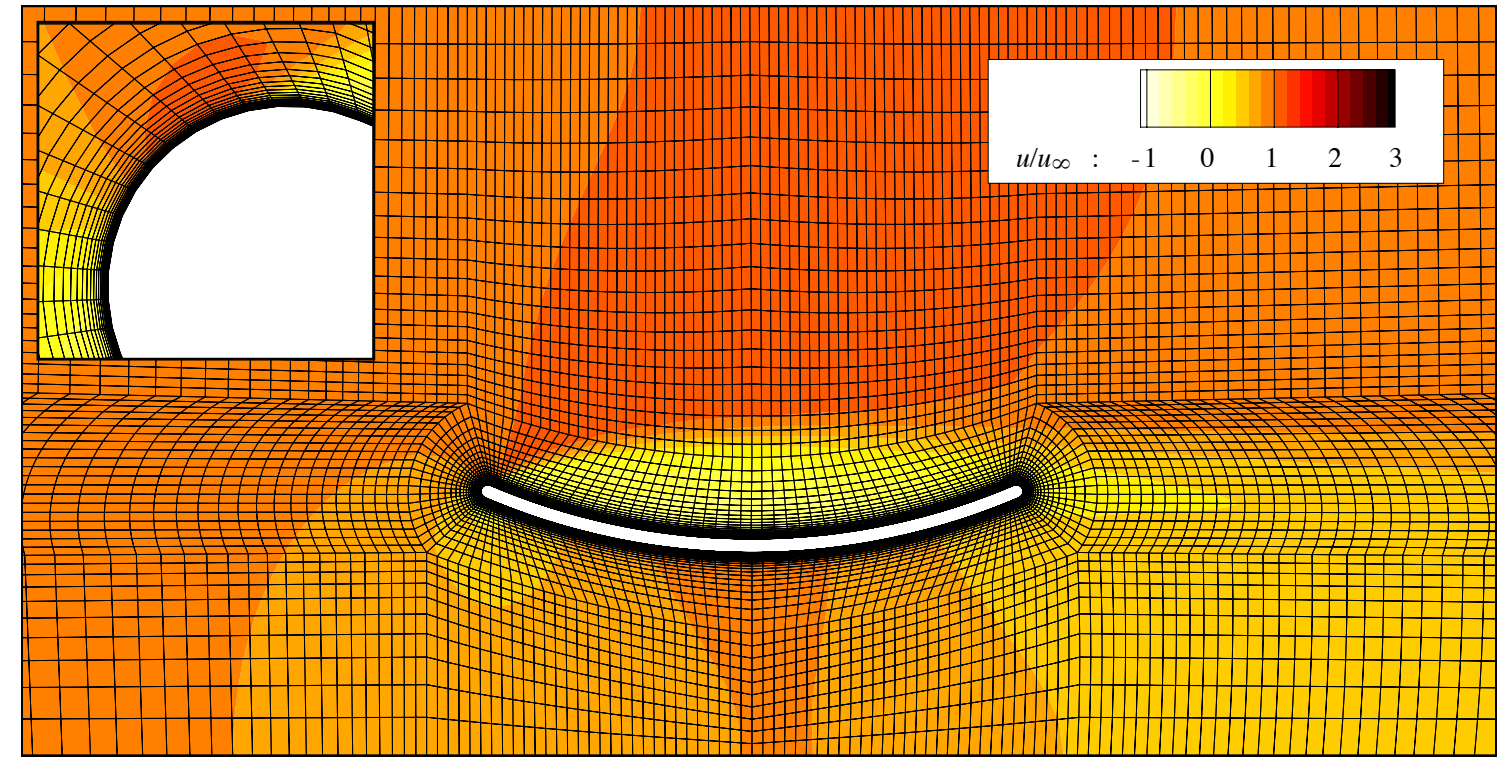

Figure 2: The mesh topology near the duct, from the axis of axial symmetry to the outer radial boundary. The domain extends $5 D$ upstream and $10 D$ downstream. Inset: the resolution at the leading edge. The contours represent streamwise velocity normalised by the upstream far field velocity. The disc momentum loss factor is $K=1$.

Table 1: Details of grids for duct $\mathrm{C}$ convergence study along with results for duct drag coefficient, $C_{D \text { duct }}$. Spatial resolution is indicated by the duct wall-normal height, $h$, and the number of elements through the domain.

\begin{tabular}{cccc}
\hline Grid & $h / D$ & $N_{\text {elem }}$ & $C_{D \text { duct }}$ \\
\hline III & $2.5 \times 10^{-5}$ & 15700 & 0.423 \\
II & $1.25 \times 10^{-5}$ & 62800 & 0.403 \\
I & $6.25 \times 10^{-6}$ & 251200 & 0.398 \\
\hline
\end{tabular}

\subsection{Spatial resolution}

The computational domain is discretised into quadrilateral elements using a structured multiblock grid. A grid convergence study is carried out on duct $\mathrm{C}$, which is expected to be most sensitive to spatial resolution due to its thin profile and high curvature. Following the method outlined by Roache [26], the same flow case is simulated on a series of successively refined grids. The computed values for duct drag, $D_{\text {duct }}$, are then compared. The coarsest grid, labelled III, is presented in figure 2. The height of the elements adjacent to the duct surface is $h=2.5 \times 10^{-5} \mathrm{D}$, and each successive layer of elements within the O-grid region grows in height by a factor of 1.2. The refined grids, II and I, have been generated by successively subdividing each element into four child elements, resulting in a linear grid refinement ratio of 2 between successive refinements.

A disc momentum loss factor of $K=1$ is chosen as the operating point for the convergence study, as preliminary computations have suggested that this point is close to peak power generation. Duct drag $D_{\text {duct }}$ is computed by the flow solver as the sum of viscous and pressure forces on the surface elements of the duct and resolved in the axial direction. It is presented as a coefficient, $C_{D \text { duct }}$, normalised by the free stream dynamic pressure and a reference area $A_{\text {ref }}$ corresponding to the total frontal area of the turbine (including the duct),

$$
C_{D \text { duct }}=\frac{D_{\text {duct }}}{\frac{1}{2} \rho u_{\infty}^{2} A_{\text {ref }}} .
$$

The three grids are described along with corresponding computed values duct drag coefficient in table 1

Profiles of non-dimensional wall distance $y^{+}=y u_{\tau} / v$ (where $u_{\tau}=\sqrt{\tau_{w} / \rho}, v$ is kinematic viscosity, $y$ is wall normal distance and $\tau_{w}$ is wall shear stress) at the surface of the duct are presented for grids I, II and III in figure 3 


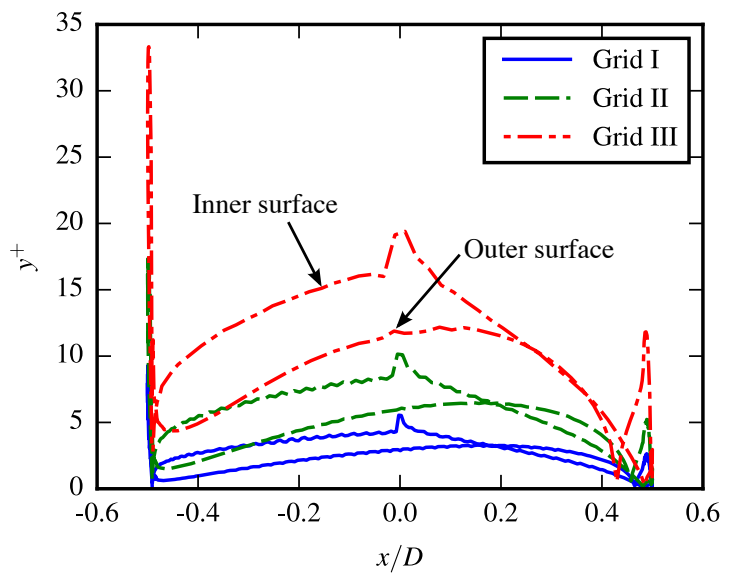

Figure 3: Profiles of non-dimensional wall distance $y^{+}$at the surface of the duct for the grids used in the convergence study.

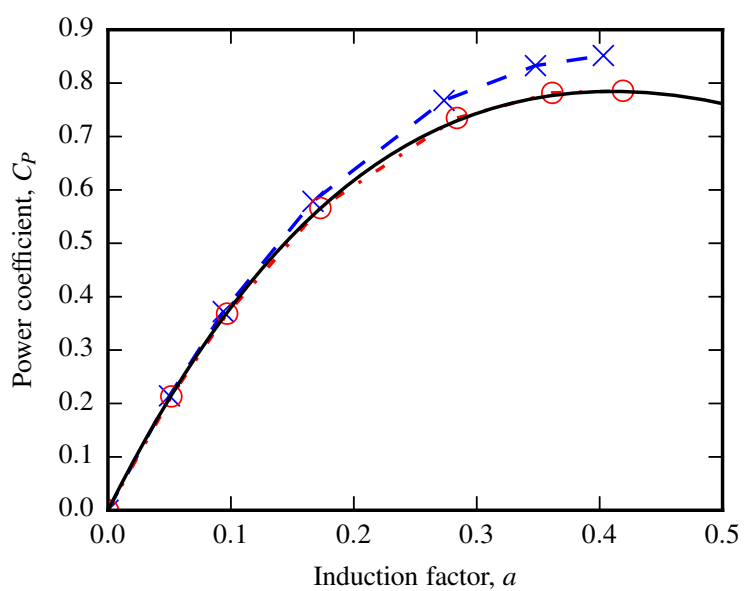

Figure 4: Comparison of computed and theoretical results for bare turbine. Solid line: theoretical result [14]; circles ( $\bigcirc)$ : computed result for low free stream turbulence from convergence study; crosses $(\times)$ : computed result for high free stream turbulence case.

Higher resolution grids $\left(y^{+}<1\right)$ were found to lead to impractically high cell counts, while lower resolution grids $\left(y^{+}>30\right)$ did not adequately resolve the geometry or shear layer at the leading edge of the duct. The errors usually associated with wall-adjacent cell heights in the range $5<y^{+}<30$ are mitigated in ANSYS Fluent by an enhanced wall treatment in the $k-\omega$ SST turbulence model [27].

The order of convergence of the set of grids is found to be $p=1.907$, close to the formal order of 2 . The corresponding grid convergence index ratio is close to unity, indicating asymptotic convergence. Grid II is chosen as the basis for the discretisation of each duct geometry in the subsequent parametric study, as it offers acceptable accuracy at a much lower computational cost than grid I.

\subsection{Actuator disc validation}

A bare disc is also simulated for comparison with the various duct geometries. The domain is discretised to a similar resolution as the medium duct grid, grid II. Predictions of power are compared with actuator disc theory for confined flow [14] in figure 4] The disc operating point is presented in terms of induction factor,

$$
a=1-\frac{u_{\mathrm{disc}}}{u_{\infty}}
$$


and performance reported in terms of the power coefficient,

$$
C_{P}=\frac{P}{\frac{1}{2} \rho A_{\text {ref }} u_{\infty}^{3}}=\frac{\Delta p A_{\text {disc }} u_{\text {disc }}}{\frac{1}{2} \rho A_{\text {ref }} u_{\infty}^{3}}
$$

where $P$ is power, and $A_{\text {ref }}$ is the reference area which is taken to be the total projected frontal area of the device (disc and duct). Note that the Betz limit of $C_{P \max }=16 / 27$ is only applicable to a rotor in unconfined flow [28]. Garrett and Cummins [14] show that in a confined flow the theoretical maximum power coefficient is greater due to the effect of the blockage ratio,

$$
C_{P \max }=\frac{16}{27} \frac{1}{(1-B)^{2}} .
$$

which has a value of 0.784 for the current conditions.

The high turbulence case is consistent with the parametric study conditions listed in section 3.1 where the inflow turbulence intensity is $T I=10 \%$. Here, the computed power coefficient is greater than the theoretical power coefficient. This result agrees qualitatively with that of Nishino and Willden [25], who show that at similar blockage ( $B=0.126)$ an increase in freestream turbulence level leads to an increase in $C_{P}$. They report that this is due to the enhanced mixing of the core and bypass flows, which reduces the bypass velocity and increases the core velocity, thereby drawing a higher volume flux through the disc for a given level of thrust. The power curve is shifted to lower induction factors, as shown in figure 4 For a more appropriate comparison with the inviscid analytical model [14], a very low freestream turbulence case $(T I=0.1 \%)$ is also shown.

\section{Results}

The set of five ducted devices and the unducted device are each simulated at up to eight operating points. Discaveraged velocity $u_{\text {disc }}$ and duct drag coefficient $C_{D}$ are monitored during each simulation, along with the flow field residuals reported by the solver. Simulations are terminated when when $u_{\mathrm{disc}}$ and $C_{D}$ change by less than $0.1 \%$ over 100 iterations, and when all flow field residuals have reached asymptotic convergence, typically requiring between $4 \times 10^{3}$ and $10^{4}$ iterations.

\subsection{Relative performance of ducted and unducted turbines}

\subsubsection{Power coefficient based on total frontal area}

Power curves for the ducted devices and an unducted device are compared in figure 5 Here the power coefficient (equation 4) is based on the total frontal area of the device, including a duct where present, so that the blockage ratio is consistent across all devices. The unducted actuator disk achieves a power coefficient of approximately $C_{P}=0.85$ (verified by comparison with theoretical result in section 3.3), whereas none of the ducted actuator disks exceed $C_{P}=0.5$.

As the power extracted from a one-dimensional flow by an actuator disc is $P=u A \Delta p$, the design intent of a cambered duct profile is to achieve a velocity increase which outweighs the corresponding elevated disc thrust and reduced disc area. This is achieved by the cambered ducts B, C, D and $\mathrm{H}$ at low disc resistance only $(K \lesssim 1)$. However at moderate and high disc thrust, the benefit of flow acceleration is diminished and the unducted device achieves a greater peak power coefficient.

\subsubsection{Power density}

Ducted turbine performance appears better when the power coefficient is based on rotor area rather than the frontal area of the whole device. Figure 6 compares the rotor-based power coefficient, also known as power density, for duct $\mathrm{C}$ and for a bare disc matching the rotor diameter (labelled 'small bare'). The power of the smaller disc is greatly increased when duct $\mathrm{C}$ is added. However a similar power increase can be achieved simply by increasing the disc diameter to match the blockage of duct C (labelled 'large bare'). 


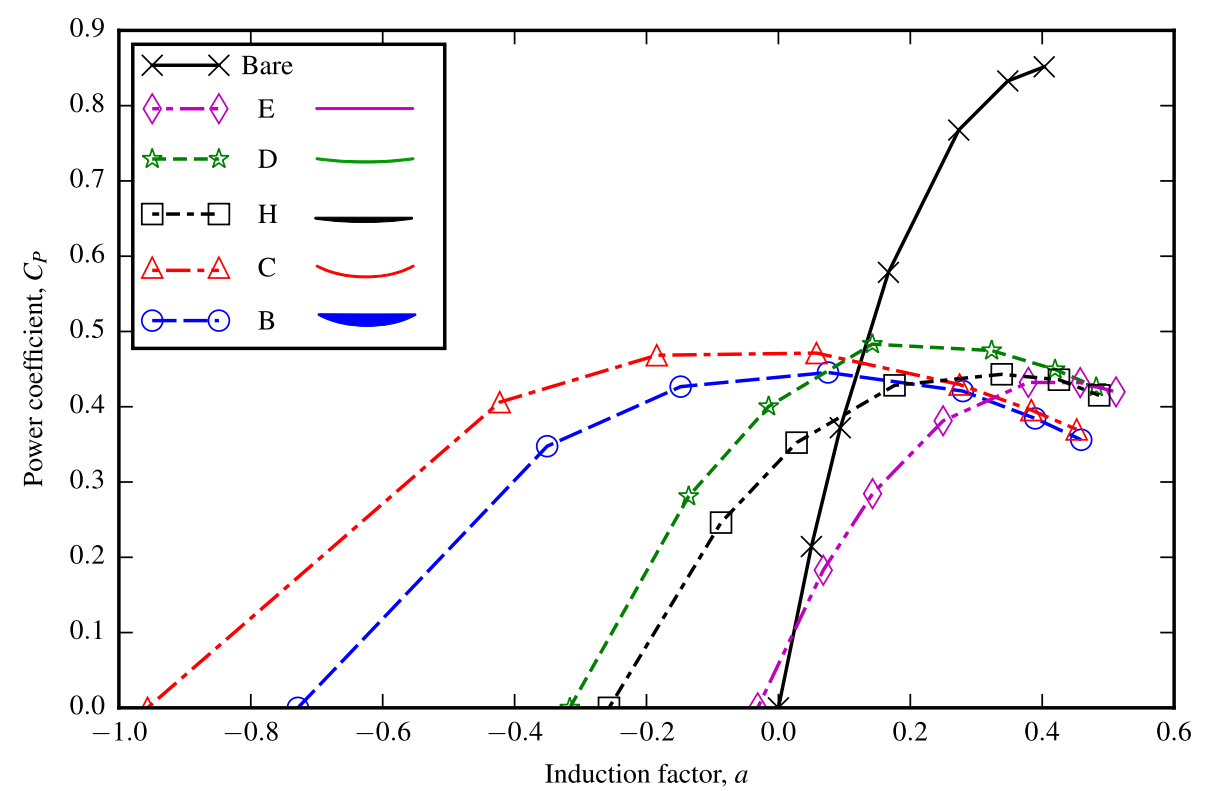

Figure 5: Comparison of power coefficient for the ducted turbines and the bare disc. The operating points correspond to momentum loss factors $K=\{0,0.25,0.5,1,2,3,4\}$, increasing from left to right.

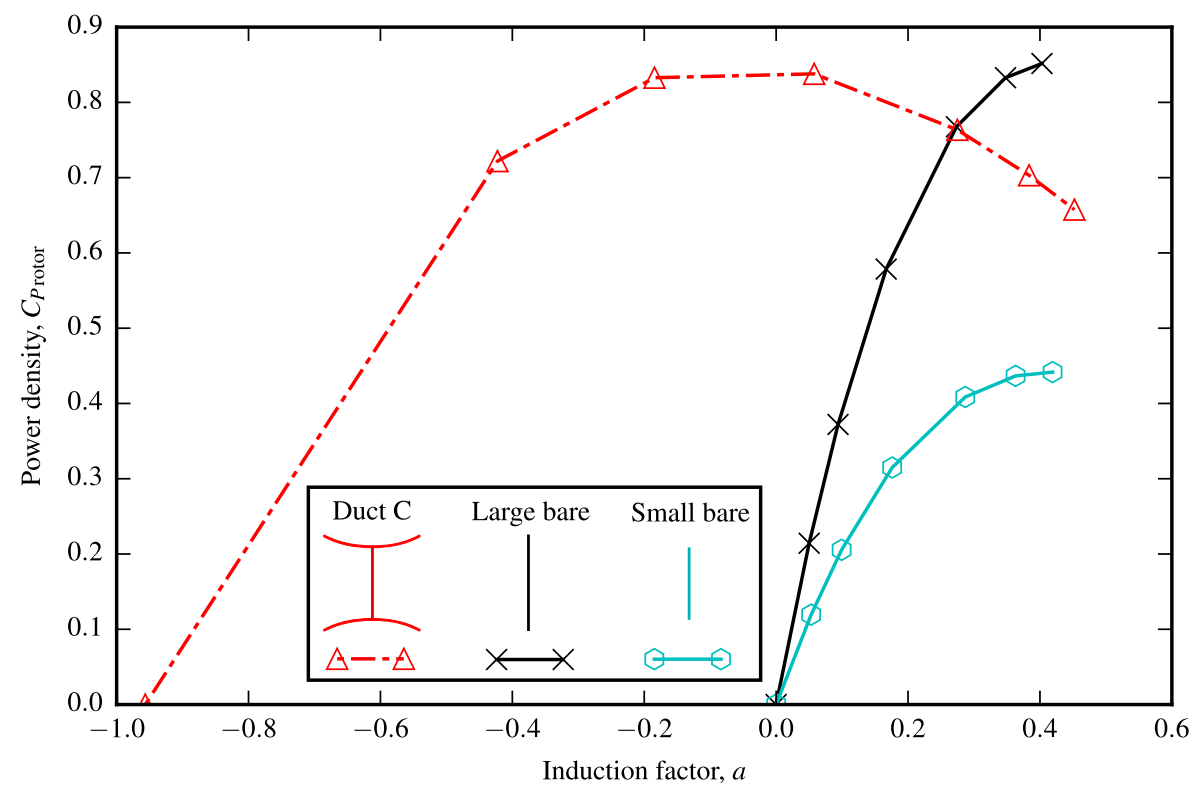

Figure 6: Comparison of rotor-based power coefficient for duct $\mathrm{C}$ with bare discs matching the inner diameter and outer diameter of the duct respectively. 


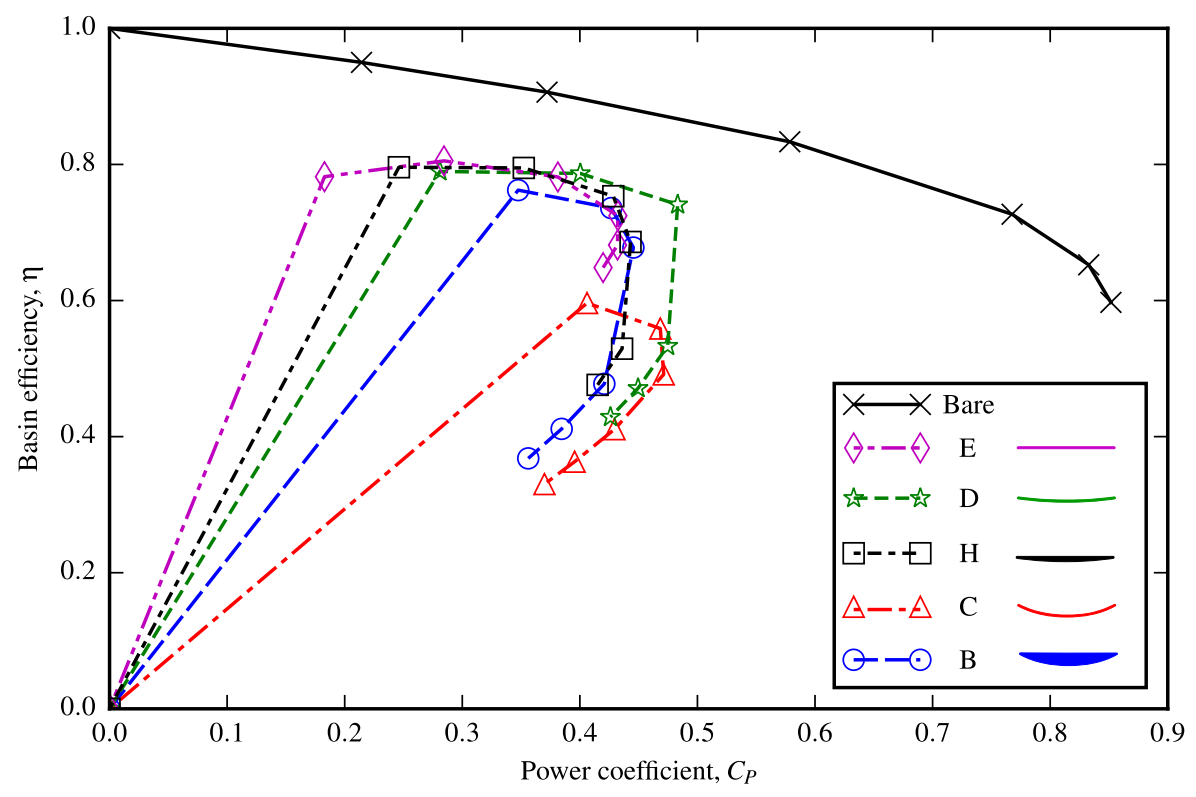

Figure 7: Comparison of efficiency for the ducted turbines and the bare disc. The operating points correspond to momentum loss factors $K=\{0$, $0.25,0.5,1,2,3,4\}$, increasing from left to right.

\subsubsection{Efficiency}

Another important performance metric for a tidal turbine is the ratio of useful power generated to total power removed from the flow, denoted 'efficiency' [29, 30]. The useful power is the mechanical power transmitted to the shaft of the turbine, in the absence of mechanical losses, calculated as $P_{\text {useful }}=T_{\text {disc }} u_{\text {disc }}=\Delta p A_{\text {disc }} u_{\text {disc }}$. The total power removed from the flow includes losses due to viscous effects such as friction drag, flow separation from the duct and wake mixing as well as the useful mechanical power. Conservation of linear momentum (see for example Shives and Crawford [30]), can be used to show that this power is given by the product of the total resistance to the flow, i.e. the thrust on the disc and duct, and the freestream velocity.

$$
P_{\text {lost }}=\left(T_{\text {disc }}+T_{\text {duct }}\right) u_{\infty}=\left(\Delta p A_{\text {disc }}+T_{\text {duct }}\right) u_{\infty}
$$

It is likely that regulators will limit the allowable change to water levels or flow speeds at a given tidal site. Any energy extracted from an open channel flow results in a reduction in water depth, whether that energy is converted to electrical power or lost to viscous action. Any unnecessary losses, such as those associated with poor hydrodynamics of the supporting structure, should be minimised.

Efficiency is presented as a function of power coefficient for each device in figure 7 It is apparent that all of the ducted discs examined are less efficient than the bare disc. In particular, a dramatic reduction in efficiency occurs for each ducted device over the range $1<K<2$, when the increased resistance of the turbine triggers an external flow separation at the leading edge of the duct [13].

\subsubsection{General effect of a duct}

The general effect of a duct on the power extracted by an actuator disc is assessed for the simplest duct geometry (E). As this duct has no camber, it does not provide any acceleration to the core flow, but nevertheless has a peak power coefficient close to the maximum achieved by the other duct geometries considered.

Noting that actuator disc power is a function of its cross-sectional area, flow velocity and local static pressure drop, longitudinal profiles of these parameters in the rotor streamtube are compared to examine the effect of the duct. Devices are compared using the merit function $f=\max \left(\eta C_{P}\right)$, reflecting the dual objectives of extracting useful mechanical power and preserving the available resource. 

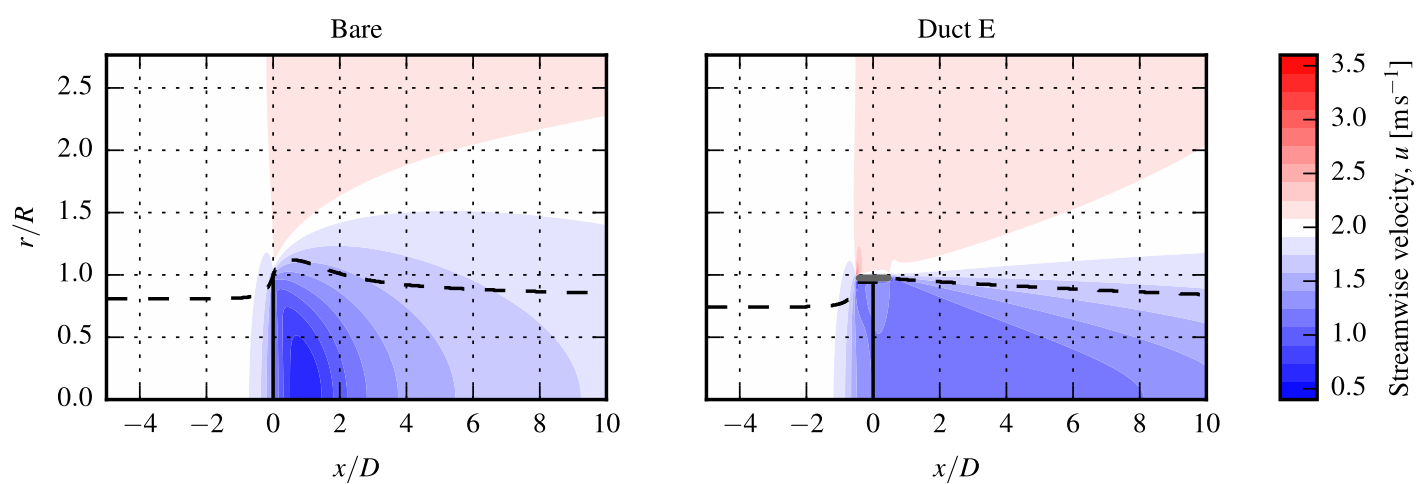

(a)
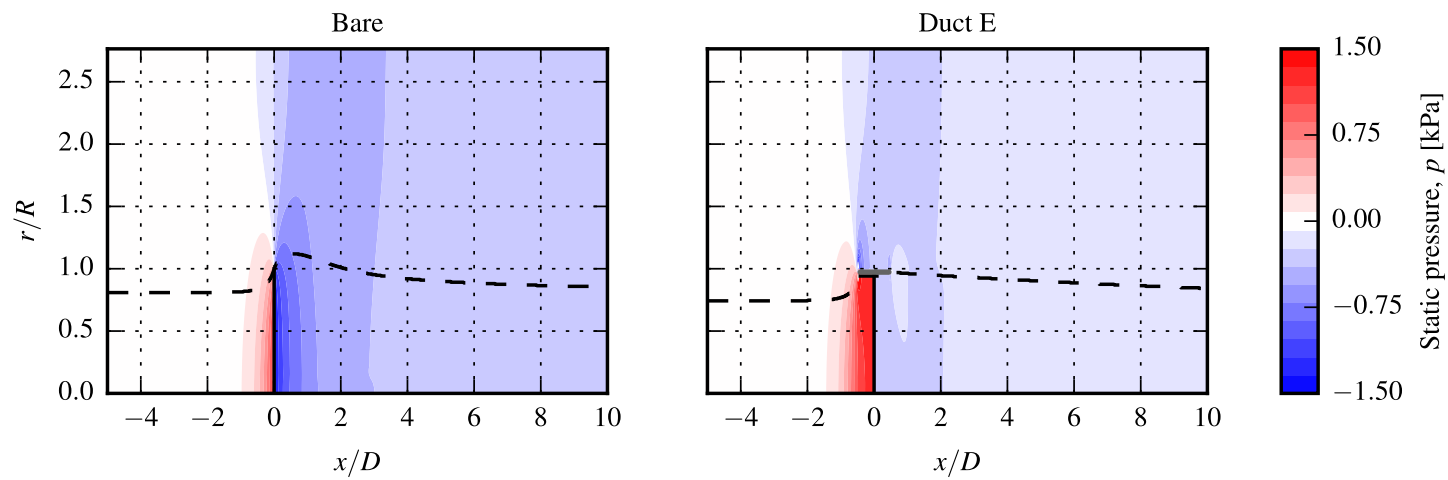

(b)

Figure 8: Contours of (a) streamwise velocity and (b) static pressure for the bare disc and duct E. The rotor streamtube is marked in each case by a broken line.

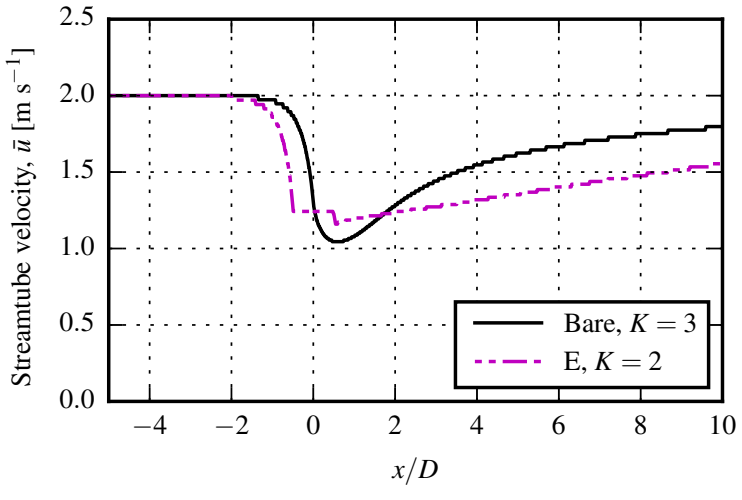

(a)

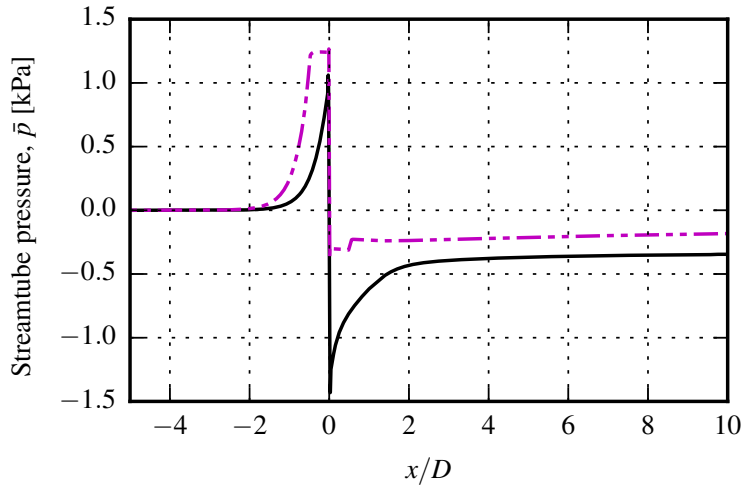

(b)

Figure 9: Variation of (a) streamtube velocity and (b) static pressure in the streamwise direction for the bare disc and duct E. 


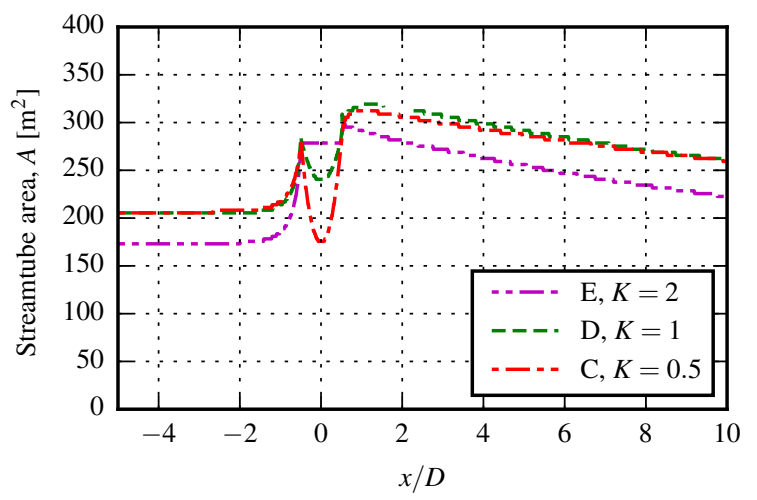

(a)

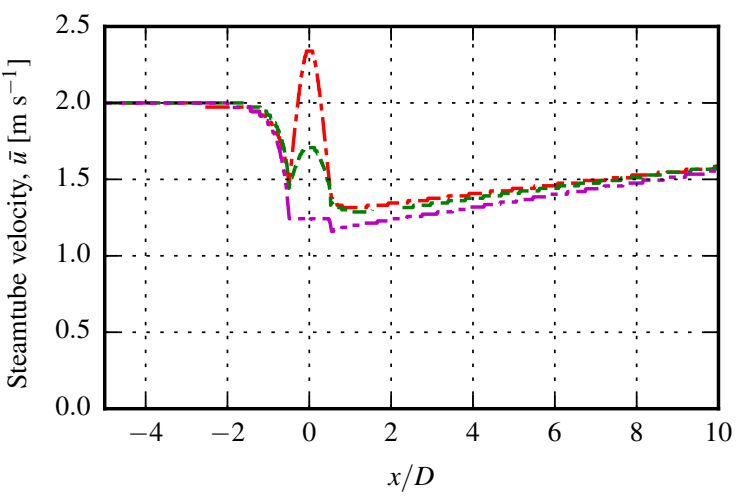

(b)

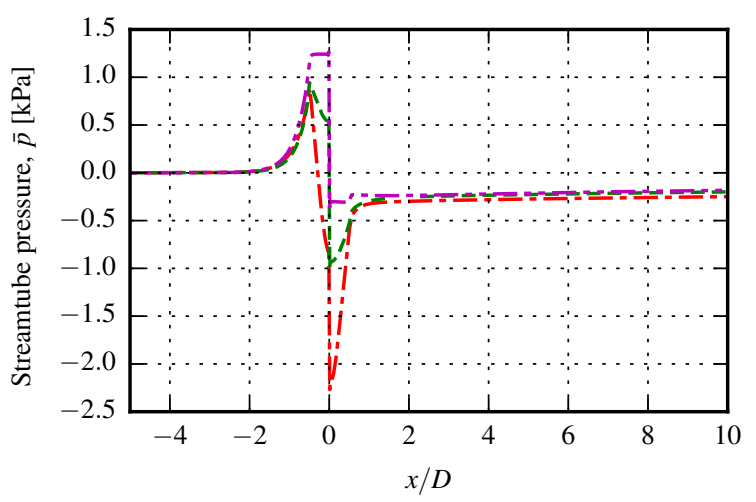

(c)

Figure 10: Variation of (a) streamtube area, (b) streamtube velocity and (c) streamtube pressure in the streamwise direction for ducts E, D and C.

Contours of streamwise velocity and static pressure for flow through the bare disc (at $K=3$ ) and duct $\mathrm{E}($ at $K=2$ ) are presented in figure 8 . In each case the streamtube intersecting the rotor tips is marked with a broken line. Spatially averaged profiles of velocity, $\bar{u}$, and static pressure, $\bar{p}$, within the rotor streamtube (referred to hereafter as streamtube velocity and streamtube pressure) are presented in figure 9 to allow direct visual comparison of ducted and unducted flows. The flow behaviour upstream of each device is quite similar: there is an increase in streamtube pressure and cross-sectional area and a decrease in velocity as the flow approaches the front of the device. However, differences arise at the turbine and in the wake.

In the unducted case, the streamtube continues to expand as it passes through the rotor, following a large reduction in static pressure across the rotor. The wake stops expanding when the pressure equilibrates between the core and bypass flows (at $x \approx 0.8 D$ ). Subsequently, turbulent mixing causes the core flow to accelerate (and shrink) and the bypass flow to decelerate, with with both tending towards a uniform downstream velocity, $u_{\infty}$.

In the ducted case, the pressure reduction is limited on the downstream side, because the flow is constrained by the duct. Free and unrestricted expansion of the flow before and aft of the rotor is prevented. In the absence of flow expansion, a pressure difference between the disc and the far wake cannot be supported. Hence the pressure change across the rotor, and consequently the extracted power, is limited.

\subsection{Effect of duct camber}

The effect of duct camber on power extraction is examined by comparing ducts $\mathrm{E}, \mathrm{D}$ and $\mathrm{C}$, at their respective optimal operating points of $K=0.5,1$ and 2. Streamwise profiles of streamtube area, bulk velocity and average static pressure are presented in figure 10. Differences in power coefficient are primarily due to disc area and velocity, as similar disc pressure changes are observed for each device. Duct $\mathrm{C}$, the most cambered device, achieves the highest flow velocity through the disc, but at the expense of disc area. The parallel duct, E, does not accelerate the flow at 


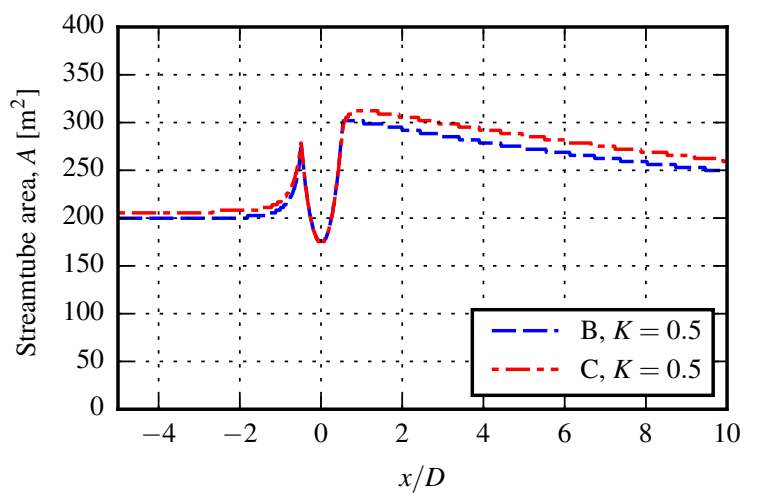

(a)

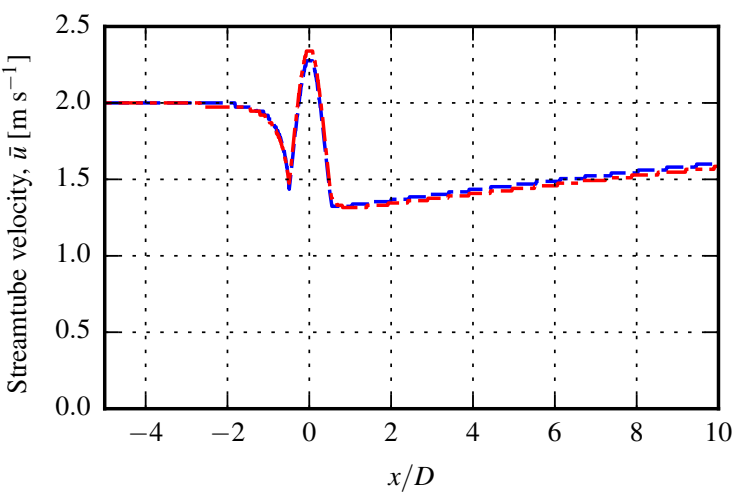

(b)

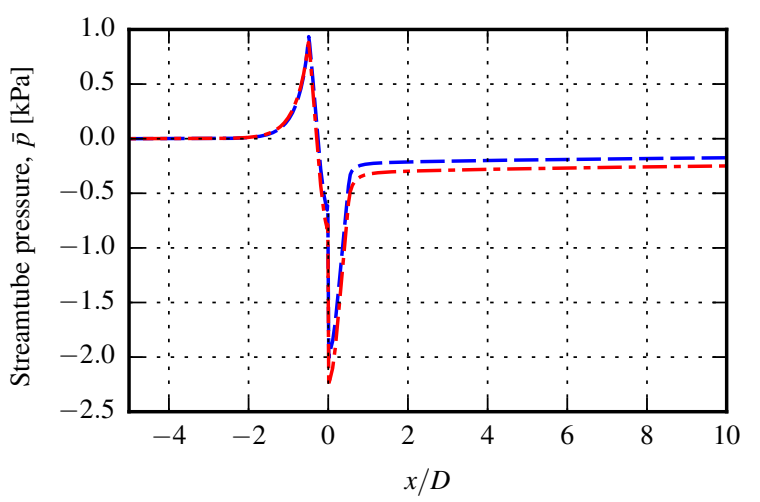

(c)

Figure 11: Variation of (a) streamtube area, (b) streamtube velocity and (c) streamtube pressure in the streamwise direction for ducts B and C.

all and has the lowest power coefficient. The best balance between flow acceleration and power-extracting area is achieved by duct D (see also figure 5), indicating that a moderate degree of camber is preferable. Note that at duct exit the curvature of ducts $\mathrm{D}$ and $\mathrm{C}$ allows a lower static pressure as greater wake expansion takes place downstream. Hence cambered ducts are able to support a greater pressure drop, and deliver higher power, than uncambered ducts.

\subsection{Effect of duct thickness}

Ducts with the same inner surface profile but different wall thicknesses (e.g. D \& H, C \& B) perform differently. The thin walled ducts $(C$ and $D)$ generally achieve higher power coefficients while the thick profiles $(B$ and $H)$ have better hydrodynamic efficiency.

Profiles of streamtube cross-sectional area, bulk velocity and cross-streamtube-averaged static pressure pressure are presented in figure 11 for ducts $\mathrm{B}$ and $\mathrm{C}$ at their optimal operating points ( $K=0.5$ in both cases). Although the disc area is identical for each device, a slightly higher disc velocity and pressure change are observed for duct $C$. This duct operates at a higher effective blockage ratio, due to a recirculation zone which develops adjacent to the concave exterior surface [13]. This feature is highlighted in figure 12, which presents contours of normalised total pressure deficit $\left(p_{0 \infty}-p_{0}\right) / \frac{1}{2} \rho u_{\infty}^{2}$ for both devices at operating points $K=0.5$ and 2 . While flow recirculation at the exterior surface of duct $\mathrm{C}$ increases its power by approximately $5 \%$ relative to duct $\mathrm{B}$ (by increasing the effective blockage ratio), it has a more significant negative impact on hydrodynamic efficiency (roughly $25 \%$ here). The location and magnitude of energy losses for ducts $\mathrm{B}$ and $\mathrm{C}$ at moderate $(K=0.5)$ and high $(K=2)$ disc resistances are visible in figure 12. The useful energy extracted by the disc is shown as a step change in total pressure. Additional energy is lost from the flow in the external recirculation region and the wake shear layer. The viscous losses in the recirculation region are much greater for duct $\mathrm{C}$ at both operating points, reducing its hydrodynamic efficiency below that of duct B. 


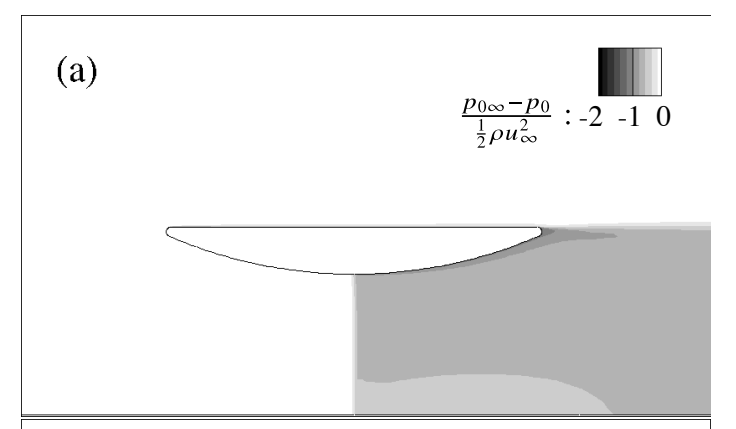

(c)

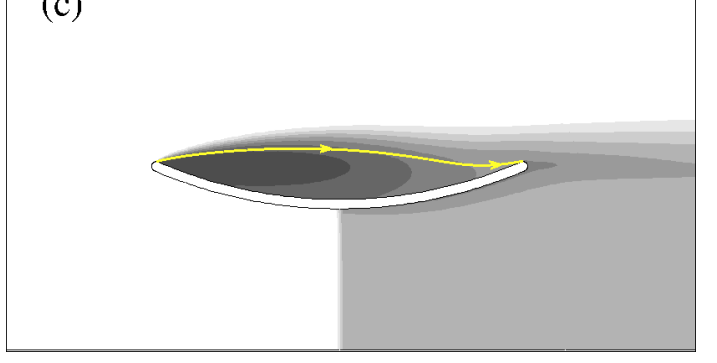

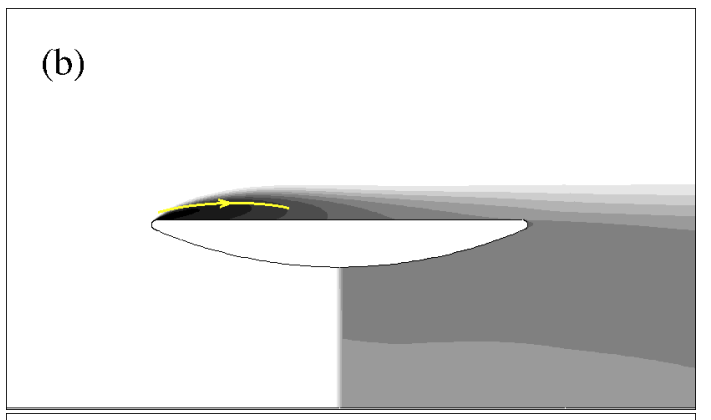

(d)

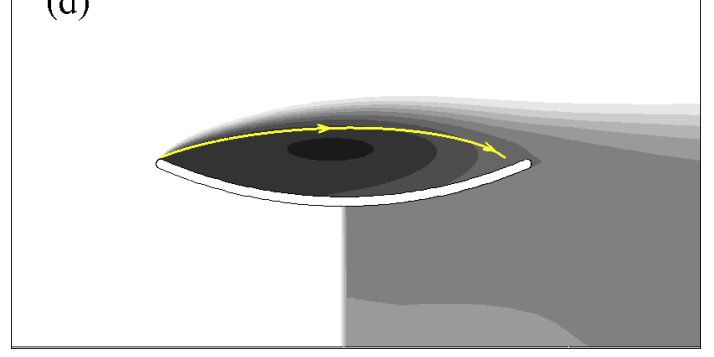

Figure 12: Contours of total pressure deficit normalised by freestream dynamic pressure, for devices B (upper row) and C (lower row) at momentum loss factors of $K=0.5$ (left hand side) and $K=2$ (right hand side). The edge of the recirculation zone is marked by a single streamline.

\section{Conclusions}

A series of bi-directional duct geometries were evaluated relative to unducted devices using a two-dimensional axisymmetric RANS solver. The rotor was modelled as an actuator disc, so that its streamwise resistance was captured, but losses associated with the centrebody, blades and swirl were not. All devices were tested with a constant external dimension that maintained an area blockage ratio of 0.131 .

An unducted disc yielded a peak power coefficient of 0.85 , whereas none of the ducted devices achieved a power coefficient of greater than 0.5 , where we define power coefficient with reference to overall device frontal area, not just rotor area. The duct was found to reduce the disc power primarily by confining the flow through the disc and limiting wake expansion. Hence the pressure difference between the disc or duct exit and the far wake was limited, resulting in a smaller permissible pressure change across the disc and ultimately lower disc power.

The addition of a duct increased power density, i.e. power coefficient based on rotor rather than device area. Therefore the power of a given rotor could be increased by augmenting it with a duct, although the overall device diameter and blockage ratio would also increase. For the conditions simulated, a similar power increase could be realised in a simpler manner by increasing the rotor diameter to match the blockage ratio of the duct-augmented alternative.

It is noted that ducts may offer other advantages for tidal turbines, such as alignment of flow, protection of blades and bearing of rotor loads, and hence beneficial design features were sought. Comparisons were made between the duct designs to identify the effects of camber and thickness.

Cambered profiles could accelerate the internal flow, but at the expense of power-extracting area. Thin, curved profiles featured a larger recirculation zone outside the duct relative to thick profiles with similar inner surface curvature. While this increased the effective blockage ratio, the resulting gain in power was outweighed by a reduction in hydrodynamic efficiency due to greater viscous losses.

The results of this study indicate that a moderate degree of camber is preferable, so that the velocity increase at the disc plane outweighs the corresponding reduction in disc area, along with a flat exterior profile to reduce viscous losses. 


\section{Acknowledgements}

The authors would like to acknowledge the use of the University of Oxford Advanced Research Computing (ARC) facility in carrying out this work. This work has been carried out as part of the PerAWaT project, commissioned by the Energy Technologies Institute.

\section{References}

[1] OpenHydro Group Ltd. (2014). [link] URL wWw . openhydro.com

[2] Lunar Energy (2014). [link] URL Www. lunarenergy . co.uk

[3] K. Foreman, B. Gilbert, A free jet wind tunnel investigation of DAWT models, rE-668, SERI/TR 01311-1 (1983).

[4] K. Foreman, A. Maciulaitis, B. Gilbert, Performance predictions and recent data for advanced DAWT models, in: ASME Solar Energy Division Meeting, 1983, p. 10.

[5] D. Phillips, P. Richards, R. Flay, CFD modelling and the development of the diffuser augmented wind turbine, WIND STRUCT INT J. 5 (2-4) (2002) 267-276.

[6] M. Shives, C. Crawford, Developing an empirical model for ducted tidal turbine performance using numerical simulation results, Proceedings of the Institution of Mechanical Engineers, Part A: Journal of Power and Energy 226 (1) (2012) $112-125$. arXiv:http://pia.sagepub.com/content/226/1/112.full.pdf+html doi:10.1177/0957650911417958

[7] C. Belloni, Hydrodynamics of ducted and open-centre tidal turbines, Ph.D. thesis, University of Oxford (2013).

[8] Atlantis Resources (2014). [link] URL http://atlantisresourcesltd.com

[9] Clean Current Power Systems Inc. (2014). [link] URL WWW.cleancurrent.com

[10] T. Setoguchi, N. Shiomi, K. Kaneko, Development of two-way diffuser for fluid energy conversion system Renewable Energy 29 (10) (2004) 1757 - 1771. doi:http://dx.doi.org/10.1016/j.renene.2004.02.007 URL http://www.sciencedirect.com/science/article/pii/S0960148104000862

[11] C. F. Fleming, S. C. McIntosh, R. H. J. Willden, Design and analysis of a bi-directional ducted tidal turbine, in: Proc. 9th European Wave and Tidal Energy Conference, Southampton, UK, 2011.

[12] C. S. K. Belloni, R. H. J. Willden, Flow field and performance analysis of bidirectional and open-centre ducted tidal turbines., in: Proc. 9th European Wave and Tidal Energy Conference, Southampton, UK, 2011.

[13] C. S. Belloni, R. H. Willden, G. T. Houlsby, A numerical analysis of bidirectional ducted tidal turbines in yawed flow, Marine Technology Society Journal 47 (4) (2013) 23-35. doi: doi:10.4031/MTSJ.47.4.16

[14] C. Garrett, P. Cummins, The efficiency of a turbine in a tidal channel, Journal of Fluid Mechanics 588 (2007) 243-251. doi:10.1017/S0022112007007781

[15] S. C. McIntosh, C. F. Fleming, R. H. J. Willden, Embedded RANS-BEM tidal turbine design, in: Proc. 9th European Wave and Tidal Energy Conference, Southampton, UK, 2011.

[16] S. Draper, G. Houlsby, M. Oldfield, A. Borthwick, Modelling tidal energy extraction in a depth-averaged coastal domain, Renewable Power Generation, IET 4 (6) (2010) 545-554. doi:10.1049/iet-rpg.2009.0196

[17] J. I. Whelan, M. R. Graham, J. Pieró, A free-surface and blockage correction for tidal turbines, J. Fluid Mech. 624 (2009) $281-291$.

[18] T. Nishino, R. H. J. Willden, The efficiency of an array of tidal turbines partially blocking a wide channel, Journal of Fluid Mechanics 708 (2012) 596-606. doi:10.1017/jfm.2012.349

[19] C. A. Consul, R. H. J. Willden, S. C. McIntosh, Blockage effects on the hydrodynamic performance of a marine cross-flow turbine, Philosophical Transactions of the Royal Society A: Mathematical, Physical and Engineering Sciences 371 (1985). doi:10.1098/rsta.2012.0299

[20] R. Malki, A. Williams, T. Croft, M. Togneri, I. Masters, A coupled blade element momentum - computational fluid dynamics model for evaluating tidal stream turbine performance, Applied Mathematical Modelling 37 (5) (2013) 3006 - 3020. doi:http://dx.doi.org/10.1016/j.apm.2012.07.025

[21] A. Mason-Jones, D. O’Doherty, C. Morris, T. O’Doherty, C. Byrne, P. Prickett, R. Grosvenor, I. Owen, S. Tedds, R. Poole, Non-dimensional scaling of tidal stream turbines, Energy 44 (1) (2012) 820 - 829. doi:10.1016/j.energy.2012.05.010

[22] ANSYS Inc., ANSYS FLUENT 14.5 User's Guide, Canonsburg, PA, USA (2012).

[23] F. R. Menter, M. Kuntz, R. Langtry, Ten years of industrial experience with the SST turbulence model, in: Turbulence, Heat and Mass Transfer 4, Begell House, CT, USA, 2003, pp. 625 - 632

[24] D. Apsley, M. Leschziner, Advanced turbulence modelling of separated flow in a diffuser, Flow, Turbulence and Combustion 63 (1-4) (2000) 81-112. doi:10.1023/A:1009930107544

[25] T. Nishino, R. H. Willden, Effects of 3-D channel blockage and turbulent wake mixing on the limit of power extraction by tidal turbines, International Journal of Heat and Fluid Flow 37 (0) (2012) 123 - 135. doi:dx.doi.org/10.1016/j.ijheatfluidflow.2012.05.002

[26] P. J. Roache, Verification and validation in computational science and engineering, Hermosa Publishers, New Mexico, 1998.

[27] ANSYS Inc., ANSYS FLUENT 14.5 Theory Guide, Canonsburg, PA, USA (2012).

[28] A. Betz, Das Maximum der theoretisch möglichen Ausnützung des Windes durch Windmotoren, Zeitschrift für das gesamte Turbinenwesen 26 (1920) 307-309.

[29] G. P. Corten, Heat generation by a wind turbine, in: 14th IEA Symposium on the Aerodynamics of Wind Turbines., ECN Rep. ECN-RX-01001,2000 , p. 7. 
[30] M. Shives, C. Crawford, Overall efficiency of ducted tidal current turbines, in: OCEANS 2010, 2010, pp. 1-6. doi:10.1109/OCEANS. 2010.5664426 
LaTeX Source Files
Click here to download LaTeX Source Files: cfleming_ducted_turbines.zip

LaTeX Source Files
Click here to download LaTeX Source Files: cfleming_ducted_turbines.zip Click here to download LaTeX Source Files: cfleming_ducted_turbines.zip

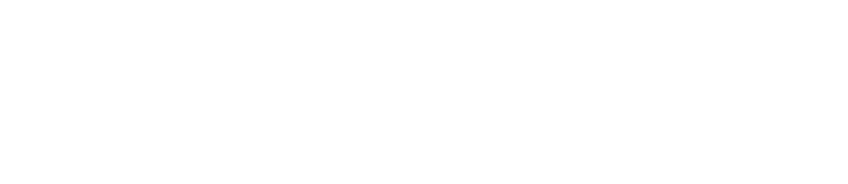

